\title{
Impact of Adalimumab on Work Productivity and Activity Impairment in Japanese Patients with Rheumatoid Arthritis: Large-Scale, Prospective, Single-Cohort ANOUVEAU Study
}

\author{
Tsutomu Takeuchi - Ryo Nakajima - Shuichi Komatsu - Kiyotaka Yamazaki · \\ Tomohiro Nakamura $\cdot$ Naoki Agata $\cdot$ Ataru Igarashi · Toshiro Tango • \\ Yoshiya Tanaka
}

Received: November 4, 2016/Published online: January 31, 2017

(c) The Author(s) 2017. This article is published with open access at Springerlink.com

\section{ABSTRACT}

Introduction: The Adalimumab Noninterventional Trial for Up-verified Effects and Utility (ANOUVEAU) was a large-scale, multicenter, prospective, observational, single-cohort study that evaluated the effects of adalimumab (ADA) on rheumatoid arthritis (RA)-related work productivity and activity impairment (RA-related WPAI) and disease activity in routine rheumatology care in Japan.

Enhanced content To view enhanced content for this article go to http://www.medengine.com/Redeem/ 3D77F0604E017A74.

T. Takeuchi $(\bowtie)$

Division of Rheumatology, Department of Internal

Medicine, Keio University School of Medicine,

Tokyo, Japan

e-mail: tsutake@z5.keio.jp

R. Nakajima $\cdot$ S. Komatsu $\cdot$ K. Yamazaki .

T. Nakamura · N. Agata

AbbVie GK, Tokyo, Japan

\section{A. Igarashi}

Department of Drug Policy and Management,

Graduate School of Pharmaceutical Sciences, The

University of Tokyo, Tokyo, Japan

T. Tango

Center for Medical Statistics, Tokyo, Japan

Y. Tanaka

The First Department of Internal Medicine, School

of Medicine, University of Occupational and

Environmental Health, Kitakyusyu, Japan
Methods: Patients with RA were categorized as paid workers (PWs, $\geq 35 \mathrm{~h} /$ week), part-time workers (PTWs, $<35 \mathrm{~h} /$ week), or homemakers (HMs, unemployed) and were administered the WPAI for RA (WPAI/RA) questionnaire. All patients who received ADA were followed for 48 weeks to evaluate safety and effectiveness.

Results: Of the 1808 patients analyzed, 825, 243, and 740 patients were PWs, PTWs, and HMs, respectively. WPAI/RA domain scores significantly improved at weeks 12,24 , and 48 in all groups, with maximum improvement observed for PWs $(p<0.05)$. Additionally, remission rates (according to Disease Activity Score 28, erythrocyte sedimentation rate, Simplified Disease Activity Index, or Health Assessment Questionnaire-Disability Index scores) and EuroQol 5-Dimension 3-Level scores significantly increased from baseline to 48 weeks in all groups $(p<0.0001)$. Analysis of patient subgroups revealed better WPAI/RA outcomes for patients who were biologic-naïve, treated with concomitant methotrexate, or with RA duration of $\leq 2$ years $(p<0.05)$. The rate of serious adverse events over 48 weeks of ADA treatment was $5.23 \%$.

Conclusions: Treatment with ADA provided sustained improvement in WPAI and had an acceptable safety profile in patients with RA.

Funding: AbbVie GK and Eisai Co., Ltd.

Trial Registration: ClinicalTrials.gov identifier, NCT01346488. 
Keywords: Patient safety; Post-marketing surveillance study; Rheumatoid arthritis; TNF inhibitor; Work productivity and activity impairment

\section{INTRODUCTION}

Rheumatoid arthritis (RA) is a systemic autoimmune disease characterized by inflammation and joint destruction [1]. The global prevalence of RA is approximately $0.24 \%$ [2]. Based on analysis of a Japanese health insurance database (Institute of Rheumatology, Rheumatoid Arthritis [IORRA], 2005 to 2011), the estimated prevalence of RA in Japan is between $0.6 \%$ and $1.0 \%$ [3].

Within 6 months of the onset of RA, patients often experience inflammatory synovitis, articular cartilage destruction, joint erosion, and consequent disability that leads to functional loss and impairment in many aspects of daily living, including work and home activities, recreation, and social relations [4-8]. Furthermore, pain associated with RA is an important predictor of sick leave and reduced productivity among employed patients with RA [9]. Physical symptoms of RA and associated functional limitations negatively impact quality of life (QoL), as demonstrated in numerous clinical trials, including one evaluating EuroQol 5-Dimension 3-Level (EQ-5D-3L) scores and their relationship with other clinical outcomes among 5284 Japanese patients with RA [10].

In addition to the personal burden RA imposes on patients and their families, RA exacts a substantial economic burden on society, particularly on employers and the healthcare system. According to an analysis of the direct medical costs and the indirect costs of care of more than 10,000 patients with RA in the IORRA database $(2007,2008)$, costs were considerable and grew with increasing disease activity and disability level or worsening QoL [11]. Much of the indirect cost associated with RA is work-related, including absenteeism (percentage of work time missed due to RA) and presenteeism (percentage of impaired work time due to RA), which are common in the first years following an RA diagnosis, even among those with mild disease $[4,12]$.

The goals of RA treatment are clinical remission or low disease activity, depending on patient-related factors [13, 14]. Proactive control of disease activity using early interventions that prevent irreversible damage may help mitigate the personal and socioeconomic burdens of this chronic disease. In addition to early treatment, combination treatment with conventional disease-modifying antirheumatic drugs (DMARDs), such as methotrexate (MTX), and biologic DMARDs, such as those targeting tumor necrosis factor- $\alpha$ (TNF- $\alpha$; infliximab, etanercept, and adalimumab [ADA; AbbVie Inc., North Chicago, IL, USA]), interleukin-6 (IL-6; tocilizumab), IL-1 (anakinra), or $\mathrm{T}$ cells (abatacept) provide favorable shortand long-term outcomes [13, 14]. Results of multiple studies (including several conducted in Japan) also indicate that TNF- $\alpha$ inhibitors such as etanercept and infliximab improve patients' employability and ability to perform work and housekeeping tasks [4, 15-20].

ADA is a human anti-TNF- $\alpha$ monoclonal antibody approved in Japan for the treatment of RA; it reduces the signs and symptoms of disease and induces a major clinical response, thus inhibiting the progression of structural damage and improving physical function in adult patients with moderate to severely active RA [21, 22]. The efficacy and safety of ADA has been demonstrated in short- and long-term international and Japanese clinical trials involving varied treatment regimens and patient populations. Results from several trials, including "Optimal Protocol for Treatment Initiation with Methotrexate and Adalimumab" (OPTIMA) and "Adalimumab, a Human Anti-TNF Monoclonal Antibody, Outcome Study for the Persistent Efficacy Under allocation to treatment strategies in early RA" (HOPEFUL 1) in Japan, demonstrated the benefits of early initiation of treatment with ADA, particularly among patients with high disease activity [23-27].

Although the efficacy and safety of ADA have been clearly established in patients with RA, including its effect on work ability, workplace and household productivity, and QoL in studies 
conducted around the world [19, 28-32], large-scale evidence of its long-term impact on work productivity and activity impairment (WPAI) in Japan is lacking $[15,33]$. This real-world Adalimumab Non-interventional Trial for Up-verified Effects and Utility (ANOUVEAU) study was conducted to evaluate the effects of ADA on RA-related work productivity and activity impairment (RA-related WPAI), as well as the association of disease activity changes on work outcomes in routine rheumatology care in Japan.

\section{METHODS}

\section{Study Design}

ANOUVEAU was a large-scale, multicenter, prospective, observational, single-cohort study conducted between May 2011 and January 2015 at 432 centers in Japan. The objectives of the study were (1) to evaluate the effect of ADA on RA-related WPAI, as well as associations between disease activity, patient characteristics, and disease duration and work outcomes; and (2) to determine changes in clinical and functional disease activity, as well as associations between these activities and disease duration. The study (ClinicalTrials.gov: NCT01346488) was approved by the Pharmaceuticals and Medical Devices Agency and was conducted in accordance with Good Post-marketing Study Practice (GPSP). Documentation was in accordance with Good Vigilance Practice/GPSP. For this reason, no ethical review by the individual facilities participating in the study was conducted. Because informed consent is not required for post-marketing observational studies that are conducted under the GPSP in Japan, the present study did not solicit informed consent from patients.

\section{Patients}

Patients were eligible for the study if they had an inadequate response to conventional therapy (e.g., conventional DMARDs or biologics other than $\mathrm{ADA}$ ) as stated in the current Japanese labeling for ADA [34, 35] and met the Japanese guidelines issued by the Japan College of Rheumatology (JCR) for the use of TNF- $\alpha$ inhibitors [36]. Patients were categorized on the basis of employment status: paid worker (PW; employed for $\geq 35 \mathrm{~h} /$ week), part-time worker (PTW; employed for $<35 \mathrm{~h} /$ week), or homemaker (HM; unemployed or employed in a capacity other than PW or PTW and able to perform basic activities of daily life [household duties, shopping, child care, exercise, and study]. Patients who were hospitalized or bedridden, had been previously treated with ADA, or were considered otherwise ineligible by investigators were excluded from the study.

\section{Treatment}

Before the initiation of ADA therapy, patients were examined for eligibility in accordance with current Japanese labeling for ADA [34, 35] and were determined to meet JCR guidelines for the use of TNF- $\alpha$ inhibitors [36]. Patients were allowed to continue prior RA treatments, such as DMARDs, glucocorticoids, and nonsteroidal anti-inflammatory drugs during the observation period. All eligible patients received ADA and were followed for 48 weeks. Patients who did not respond to therapy and were not receiving DMARDs (including MTX) were allowed a dose adjustment to $80 \mathrm{mg}$ every other week. If a patient withdrew from the study, the date and reason for discontinuation were recorded. Reasons for discontinuation of ADA treatment included insufficient efficacy and occurrence of adverse events.

\section{Assessments and Outcomes}

Patient demographics and characteristics were recorded in a case report form at baseline, and patients were administered validated questionnaires at baseline and at weeks 12, 24, 36 , and 48. Work-related outcomes were measured using the Work Productivity and Activity Impairment questionnaire for RA (WPAI/RA [Japanese-Japan $\mathrm{v}$ 2.1]), which comprises four domains: absenteeism, 
presenteeism, percentage of overall work impairment (OWI) due to RA, and percentage of activity impairment (AI) due to RA. For HMs, only AI data were obtained. Absenteeism was calculated as significance level (two-sided) with a power of 0.90 . To ensure 500 eligible PWs and 500 eligible HMs, enrollment targets were 1000 patients for each group, assuming a 50\% withdrawal rate. Data were analyzed using the last observation

$$
\text { Absenteeism }=\frac{[\text { hours absent from work due to RA }]}{[\text { hours absent from work due to RA }+ \text { hours actually worked }]} \times 100 \% \text {. }
$$

Missed work days per year were calculated from WPAI/absenteeism. Presenteeism was calculated using a visual analog scale (VAS) as the percentage of reduction in productivity while working due to RA. OWI was calculated as

$$
\begin{aligned}
\text { OWI }= & \text { Absenteeism } \\
& +[(1-\text { absenteeism }) \times \text { presenteeism }] \\
& \times 100 \% .
\end{aligned}
$$

AI was assessed using a VAS as a percentage of impairment due to RA in regular daily activities other than working at a job.

Disease activity, including functional and clinical response, was assessed using the Health Assessment Questionnaire-Disability Index (HAQ-DI) for RA; Disease Activity Score based on 28 joints and erythrocyte sedimentation rate (DAS28ESR); Disease Activity Score based on 28 joints and C-reactive protein (DAS28CRP); Clinical Disease Activity Index (CDAI); Simplified Disease Activity Index (SDAI) [14]; and EQ-5D-3L (descriptive sections: mobility, self-care, usual activities, pain-discomfort, and anxiety-depression).

Safety was assessed throughout the study by recording adverse events, including abnormal laboratory findings, in the case report form.

\section{Statistical Analysis}

Sample size calculations were based on results of a previous study conducted in Germany [37] in which 469 RA patients were deemed necessary to detect an average difference of 5 days (absence from work for an average of 21 days) at the 0.05 carried forward (LOCF) method when there were missing values before week 48 and are presented as mean $\pm \mathrm{SD}$ unless indicated otherwise. To compare differences between groups at baseline, the Chi square test was used for categorical variables, and the Wilcoxon rank sum test or Kruskal-Wallis test was used for continuous variables. For WPAI/RA scores, the Wilcoxon signed-rank test was used to compare baseline scores with scores at 12, 24, and 48 weeks; the Wilcoxon rank sum test or Kruskal-Wallis test was used to compare the differences in the changes from baseline between groups. Absenteeism was also assessed in a subgroup of patients with baseline absenteeism $>0$ days. The Fisher exact test was used to compare remission rates at baseline and at 12 , 24 , and 48 weeks. The Wilcoxon signed-rank test was used to compare EQ-5D-3L at baseline and at 12,24 , and 48 weeks. For regression coefficients, Pearson product-moment was used to examine the correlations between WPAI/RA domains (absenteeism, presenteeism, OWI, and AI) and clinical responses (DAS28CRP, SDAI, HAQ-DI, and EQ-5D-3L). PWs were stratified on the basis of their baseline characteristics (biologic use, MTX use, MTX dose, RA duration, and age) to evaluate the effect of baseline characteristics on WPAI/RA domains at week 48 . The Wilcoxon signed-rank test was used to compare differences between baseline and 48 weeks, and the Wilcoxon rank sum test was used to compare the differences between subgroups. SAS ${ }^{\circledR} 9.2$ (SAS Institute Inc., Cary, NC, USA) was used for statistical analysis. 


\section{RESULTS}

\section{Patient Disposition and Clinical Characteristics}

Of the 1973 patients enrolled in this study, 1968 and 1808 were eligible for safety and effectiveness analyses, respectively. Most cases of ineligibility were due to a lack of clinical data. Of the 1808 patients that were evaluable for efficacy analyses, 825, 243, and 740 were PWs, PTWs, and HMs, respectively. The groups were significantly different with respect to age $(p<0.0001)$; HMs were older (mean [SD], 62.2 [12.6] years) than PWs (49.9 [11.7] years) and PTWs (53.9 [11.8] years). PTWs and HMs were predominantly women. The overall disease duration of RA was longer in HMs (8.6 [9.2] years) than PWs (5.0 [6.2] years) and PTWs (6.1 [7.1] years), with significant group differences $(p<0.0001)$. Additionally, baseline characteristics varied among the three groups in terms of stage of RA, class of RA, MTX dose, ESR, CRP, Patient Global Assessment (PtGA)/VAS, Physician Global Assessment (PhGA)/VAS, DAS28ESR, DAS28CRP, HAQ-DI, EQ-5D-3L, and AI scores (Table 1). In all groups, the majority of patients belonged to RA stages I and II and RA classes I and II.

\section{Changes in WPAI/RA Domain Scores}

Based on the estimation from WPAI/ absenteeism results, missed work days per year in PWs $(n=677)$ decreased significantly from $7.48 \pm 19.64$ (mean \pm SD) at baseline to $3.97 \pm 14.81$ at the final assessment $(p<0.0001)$. A total of 525/677 PWs (77.5\%) and 134/180 PTWs (74.4\%) had a baseline absenteeism of 0 days. Among patients with baseline absenteeism of $>0$ days, there was a similar decrease $(p<0.0001)$ in absenteeism in the PW $(n=152)$ and PTW $(n=46)$ groups over 48 weeks (Fig. 1a). Relative to baseline, the remaining WPAI/RA domain scores also significantly decreased at all time points in all study groups $(p<0.001$ for all comparisons; Fig. 1b-d). Reduction in absenteeism and AI did not differ between groups, whereas improvements in presenteeism and OWI were greater in the PW than the PTW group at all time points $(p<0.05)$. This was true except at week 48 , when the group differences indicated marginal improvements $(p=0.0579$ for presenteeism, $p=0.0808$ for OWI).

\section{Achievement of Remission at Week 48}

The proportion of patients achieving remission according to DAS28CRP, SDAI, or HAQ-DI scores significantly increased at 12,24 , and 48 weeks compared with baseline in all groups $(p<0.0001$ for all comparisons; Fig. 2). Although the proportion of patients in HAQ-DI remission was higher at baseline in the PW (44.8\%) and PTW (40.6\%) groups in comparison to the HM group (27.7\%), there was a similar increase in the proportion of patients achieving remission over 48 weeks in all groups $(p<0.0001$; Fig. 2).

\section{Change in QoL}

The improvement in disease activity as assessed by DAS28CRP, SDAI, or HAQ-DI scores was also reflected in the significant improvement in QoL assessed by EQ-5D-3L scores in all groups through week 48, with differences from baseline occurring at all time points ( $p<0.0001$ for all comparisons; Fig. 2).

\section{Correlation with WPAI/RA Domain Scores and Clinical Responses at Week 48}

There was a trend toward a positive linear correlation between improvement in WPAI/RA domain scores and decrease in disease activity, as assessed by DAS28CRP, SDAI, and HAQ-DI scores at week 48, except absenteeism (DAS28CRP and SDAI) in PTWs. There was a trend toward a negative linear correlation between improvements in WPAI/RA domain scores and increases in EQ-5D-3L for all groups except absenteeism in PTWs $(p<0.0001$; Table 2). 
Table 1 Baseline demographics and clinical characteristics

\begin{tabular}{|c|c|c|c|}
\hline Characteristic & $\begin{array}{l}\text { PW (employed } \\
\geq 35 \text { h/week) }\end{array}$ & $\begin{array}{l}\text { PTW (employed } \\
<35 \mathrm{~h} / \text { week) }\end{array}$ & $\begin{array}{l}\text { HM } \\
\text { (unemployed) }\end{array}$ \\
\hline 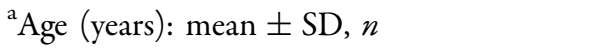 & $49.9 \pm 11.7,825$ & $53.9 \pm 11.8,243$ & $62.2 \pm 12.6,740$ \\
\hline${ }^{\mathrm{a}}$ Women: $n(\%)$ & $523(63.4)$ & $219(90.1)$ & $629(85)$ \\
\hline $\begin{array}{l}{ }^{\mathrm{a}} \mathrm{RA} \text { duration (years): mean } \pm \mathrm{SD}, n \\
{ }^{\mathrm{a}, \mathrm{f}} \text { RA stage: } n(\%)\end{array}$ & $5.0 \pm 6.2,785$ & $6.1 \pm 7.1,229$ & $8.6 \pm 9.2,688$ \\
\hline I & $289(35.2)$ & $71(29.3)$ & $137(18.6)$ \\
\hline II & $304(37.0)$ & $82(33.9)$ & $231(31.3)$ \\
\hline III & $146(17.8)$ & $48(19.8)$ & $194(26.3)$ \\
\hline IV & $83(10.1)$ & $41(16.9)$ & $175(23.7)$ \\
\hline \multicolumn{4}{|l|}{ a,g RA class: $n(\%)$} \\
\hline I & $269(32.7)$ & $61(25.1)$ & $136(18.4)$ \\
\hline II & $485(58.9)$ & $162(66.7)$ & $444(60.0)$ \\
\hline III & $66(8.0)$ & $19(7.8)$ & $149(20.1)$ \\
\hline IV & $3(0.4)$ & $1(0.4)$ & $11(1.5)$ \\
\hline${ }^{\mathrm{a}} \mathrm{MTX}$ dose (mg/week): mean $\pm \mathrm{SD}, n$ & $10.0 \pm 3.9,761$ & $9.4 \pm 2.9,224$ & $9.0 \pm 3.1,631$ \\
\hline${ }^{\mathrm{a}} \operatorname{ESR}(\mathrm{mm} / \mathrm{h}):$ mean $\pm \mathrm{SD}, n$ & $32.8 \pm 26.7,663$ & $38.5 \pm 28.8,202$ & $44.2 \pm 30.0,606$ \\
\hline${ }^{\mathrm{a}} \mathrm{CRP}(\mathrm{mg} / \mathrm{dL}):$ mean $\pm \mathrm{SD}, n$ & $1.67 \pm 3.10,796$ & $1.50 \pm 2.15,240$ & $1.84 \pm 2.37,714$ \\
\hline TJC (0-28): mean $\pm S D, n$ & $5.7 \pm 5.6,797$ & $5.8 \pm 5.5,240$ & $5.9 \pm 5.7,722$ \\
\hline SJC (0-28): mean $\pm S D, n$ & $5.7 \pm 5.3,797$ & $6.1 \pm 5.0,239$ & $5.6 \pm 5.0,722$ \\
\hline${ }^{\mathrm{a}} \mathrm{PtGA} / \mathrm{VAS}(0-100 \mathrm{~mm}):$ mean $\pm \mathrm{SD}, n$ & $48.1 \pm 26.1,795$ & $49.5 \pm 25.3,241$ & $53.3 \pm 26.0,722$ \\
\hline${ }^{\mathrm{a}} \mathrm{PhGA} / \mathrm{VAS}(0-100 \mathrm{~mm}):$ mean $\pm \mathrm{SD}, n$ & $46.2 \pm 22.8,778$ & $49.3 \pm 22.7,231$ & $50.2 \pm 23.0,708$ \\
\hline${ }^{\mathrm{a} D A S 28 E S R:}$ mean $\pm \mathrm{SD}, n$ & $4.6 \pm 1.4,653$ & $4.8 \pm 1.3,197$ & $5.0 \pm 1.3,600$ \\
\hline${ }^{\mathrm{a} D A S 28 C R P}:$ mean $\pm \mathrm{SD}, n$ & $4.1 \pm 1.3,779$ & $4.2 \pm 1.2,234$ & $4.3 \pm 1.2,704$ \\
\hline SDAI: mean $\pm \mathrm{SD}, n$ & $22.5 \pm 13.5,766$ & $23.1 \pm 13.0,225$ & $23.7 \pm 12.7,690$ \\
\hline CDAI: mean $\pm \mathrm{SD}, n$ & $20.8 \pm 12.4,775$ & $21.7 \pm 12.1,230$ & $22.0 \pm 12.0,705$ \\
\hline${ }^{\mathrm{a}} \mathrm{HAQ}-\mathrm{DI}:$ mean $\pm \mathrm{SD}, n$ & $0.750 \pm 0.652,761$ & $0.828 \pm 0.650,224$ & $1.165 \pm 0.806,676$ \\
\hline${ }^{\mathrm{a}} \mathrm{EQ}-5 \mathrm{D}-3 \mathrm{~L}:$ mean $\pm \mathrm{SD}, n$ & $0.654 \pm 0.155,756$ & $0.638 \pm 0.138,218$ & $0.597 \pm 0.154,663$ \\
\hline${ }^{\mathrm{b}}$ Absenteeism (\%): mean $\pm \mathrm{SD}, n$ & $7.5 \pm 19.6,677$ & $11.1 \pm 23.5,180$ & NA \\
\hline${ }^{\mathrm{c}}$ Presenteeism (\%): mean $\pm \mathrm{SD}, n$ & $41.1 \pm 29.1,726$ & $38.6 \pm 28.6,197$ & NA \\
\hline${ }^{\mathrm{d}} \mathrm{OWI}(\%):$ mean $\pm \mathrm{SD}, n$ & $42.3 \pm 29.8,665$ & $42.0 \pm 31.3,178$ & NA \\
\hline
\end{tabular}


Table 1 continued

\begin{tabular}{llll}
\hline Characteristic & $\begin{array}{l}\text { PW (employed } \\
\mathbf{3} \text { h/week) }\end{array}$ & $\begin{array}{l}\text { PTW (employed } \\
<35 \text { h/week) }\end{array}$ & $\begin{array}{l}\text { HM } \\
\text { (unemployed) }\end{array}$ \\
\hline${ }^{\mathrm{a}, \mathrm{e}}$ AI $(\%):$ mean $\pm \mathrm{SD}, n$ & $44.2 \pm 28.4,759$ & $47.9 \pm 28.2,219$ & $54.3 \pm 26.7,653$ \\
\hline
\end{tabular}

Some data for almost all characteristics (except for age and sex) were missing; therefore, the evaluation is based on available data only

$A I$ activity impairment, $C D A I$ Clinical Disease Activity Index (to assess clinical response), $C R P$ C-reactive protein, $D A S 28 C R P$ Disease Activity Score based on 28 joints and CRP, DAS28ESR Disease Activity Score based on 28 joints and ESR, EQ-5D-3L EuroQol 5-Dimension 3-Level, ESR erythrocyte sedimentation rate, HAQ-DI Health Assessment Questionnaire-Disability Index, $H M$ homemaker, $M T X$ methotrexate, $N A$ data not available, OWI overall work impairment, $P h G A / V A S$ physician global assessment using VAS, $P t G A / V A S$ patient global assessment using VAS, PTW part-time worker, $P W$ paid worker, $R A$ rheumatoid arthritis, $S D$ standard deviation, $S D A I$ Simplified Disease Activity Index (to assess functional response), $S J C$ swollen joint count, TJC tender joint count, $V A S$ visual analog scale

a Significant differences $(p<0.05)$ existed between the comparison groups in terms of these baseline characteristics. Statistical significance was calculated using a Chi square test for categorical variables and a Wilcoxon rank sum test or Kruskal-Wallis test for continuous variables

b Absenteeism: percentage of work time missed due to RA

c Presenteeism: percentage of impairment while working due to RA (calculated using a numerical rating scale)

d OWI (\%): percentage of overall work impairment due to $\mathrm{RA}=[$ absenteeism $+\{(1-$ absenteeism $) \times$ presenteeism $\}] \times$ $100 \%$

e AI (\%): percentage of impairment in daily activities (other than work) due to RA (assessed using a VAS)

f Stage of RA: I, early; II, moderate; III, severe; and IV; end stage [38]

g Class of RA: I, complete functional capacity with ability to carry on all usual duties without handicaps; II, functional capacity adequate to conduct normal activities despite handicap of discomfort or limited mobility of one or more joints; III, functional capacity adequate to perform only few or none of the duties of usual occupation or of self-care; IV, largely or wholly incapacitated with patient bedridden or confined to wheelchair, permitting little or no self-care [38]

\section{Association Between Change in WPAI/RA Domain Scores and Baseline Characteristics in PW Group}

There was a significant improvement in WPAI/ RA domain scores at week 48 in biologic-naïve patients compared with biologic-experienced patients ( $p<0.05$; Table 3$)$. Concomitant use of MTX has been shown to increase the efficacy of ADA treatment, decreasing RA disease activities more efficiently than ADA alone [39]. Along these lines, patients treated with concomitant MTX versus those treated without MTX showed greater improvement in presenteeism (19.1 vs. 9.8), OWI (19.4 vs. 7.5), and AI (22.4 vs. 12.9$)$ $(p<0.05)$. Additionally, when initiated at an early stage of RA, biologics treatment shows higher efficiency to reduce disease activity [40]. Consistent with this notion, patients with $\leq 2$ years of RA duration exhibited significantly improved AI compared to those with an RA duration of $>2$ years $(p<0.05)$.

\section{Safety}

The safety profile of ADA in the present study was consistent with previous reports [39, 41]. The rate of serious adverse events over 48 weeks of ADA treatment was $5.23 \%$ (103/1968 cases), with infections and infestations being the most commonly reported adverse events (Table 4). Other common serious adverse events were neoplasms (benign, malignant, and unspecified [including cysts and polyps]), which were reported in 14 patients (17 events [0.9\%]), followed by respiratory, thoracic, and mediastinal disorders, which were reported in 11 patients (14 events [0.7\%]).

\section{DISCUSSION}

The efficacy and safety of ADA in patients with RA is well established; however, its impact on work productivity and activity impairment in 
a

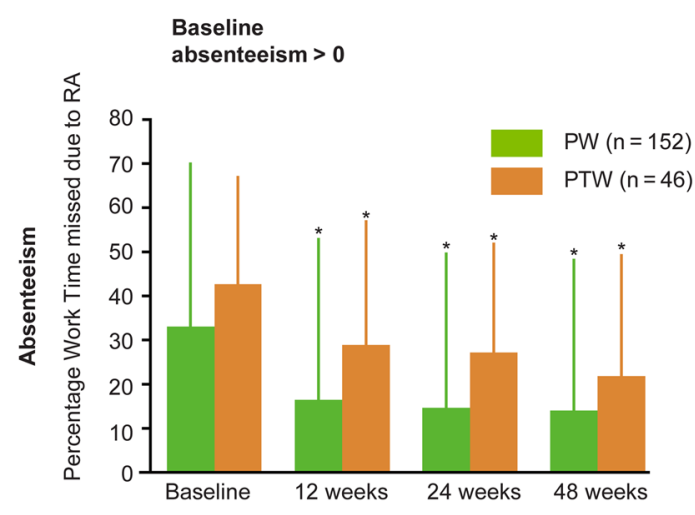

c

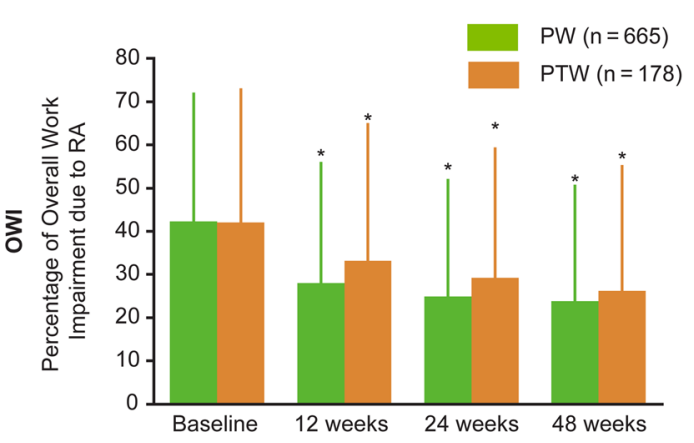

Fig. 1 Change from baseline in WPAI/RA domain scores. a Patients with baseline absenteeism $>0$ days, b presenteeism, c OWI, and d AI. Only patients with absenteeism $>0$ are shown in a, whereas all eligible RA patients are included in $\mathbf{b}-\mathbf{d}$. $A I$ activity impairment, $H M$ homemaker, unemployed, $O W I$ overall work impairment, $P T W$ part-time worker employed for $<35 \mathrm{~h} /$ week, $P W$ paid worker employed for $\geq 35 \mathrm{~h} /$ week, $R A$ rheumatoid arthritis,

b

Presenteeism
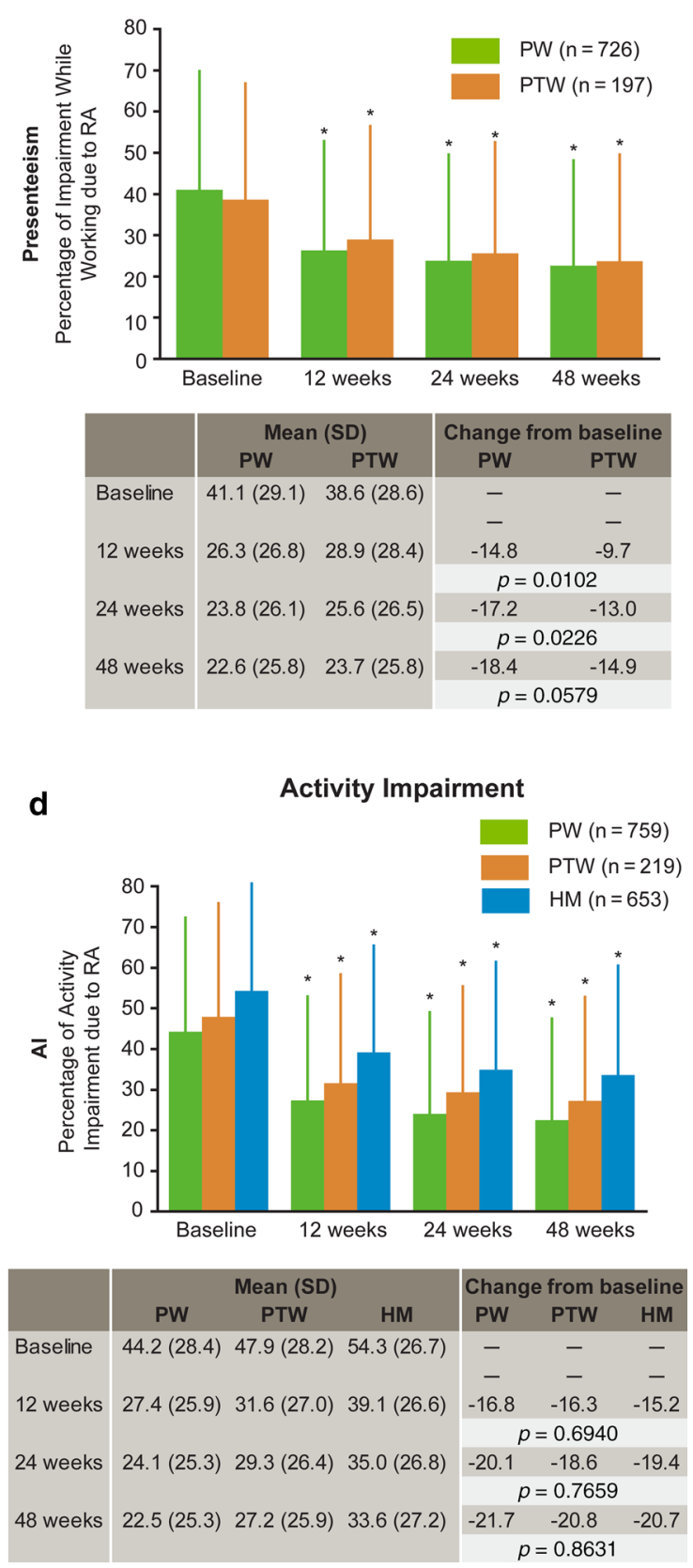

$S D$ standard deviation, WPAI work productivity and activity impairment. ${ }^{*} p<0.0001$ compared to the corresponding baseline values. The Wilcoxon signed-rank test was used to compare baseline and 12, 24, and 48 weeks, and the Wilcoxon rank sum test or the Kruskal-Wallis test was used to compare the difference in the changes from baseline between the two groups. Data were analyzed using the last observation carried forward method 

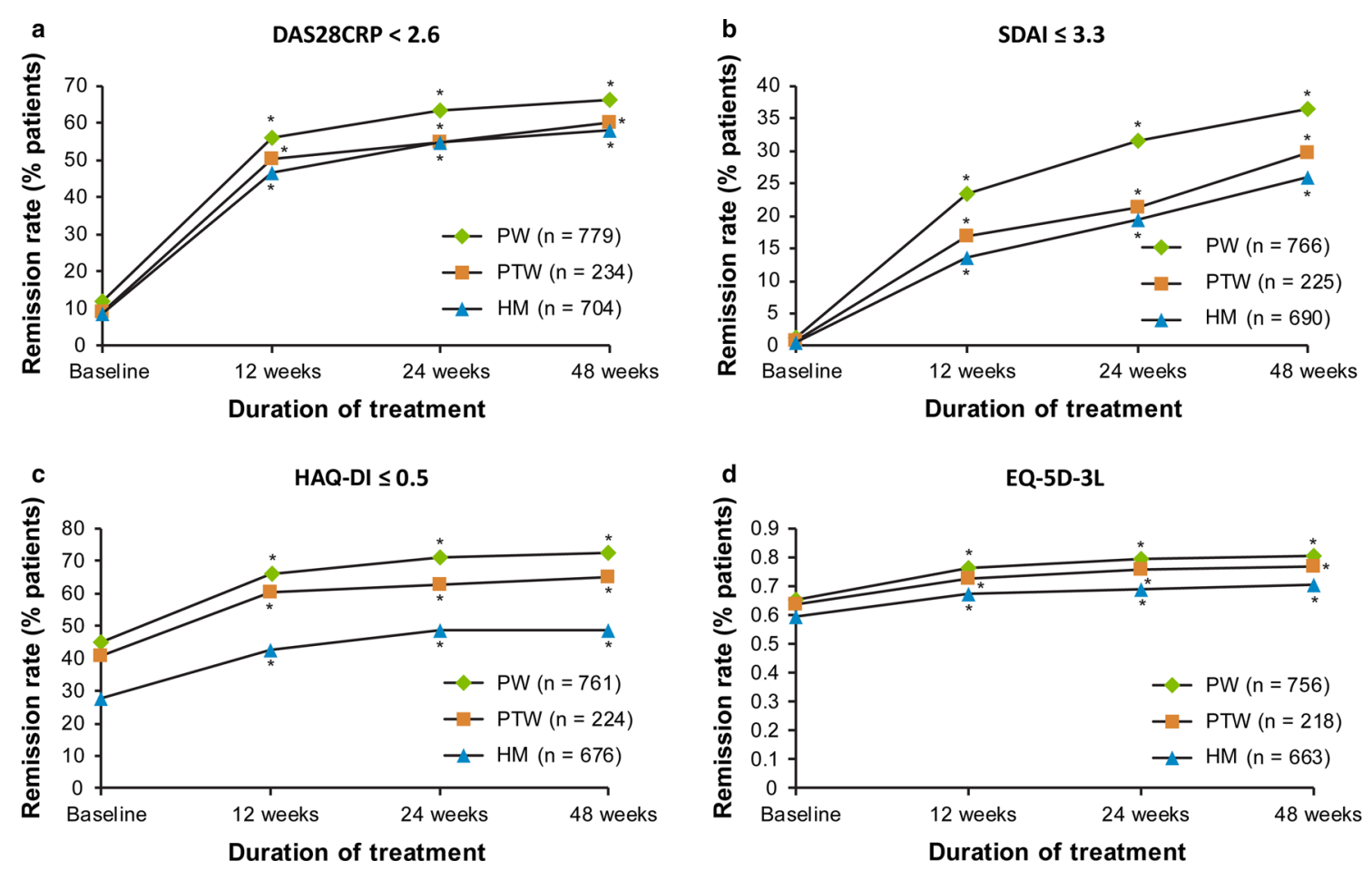

Fig. 2 Remission rates based on DAS28CRP, SDAI, and HAQ-DI along with EQ-5D-3L scores. CRP C-reactive protein, DAS28CRP Disease Activity Score based on 28 joints and CRP, EQ-5D-3L EuroQol 5-Dimension 3-Level, HAQ-DI Health Assessment Questionnaire-Disability Index, $H M$ homemaker, $P T W$ part-time worker, $P W$ paid worker,

Japan has not been studied until now. ANOUVEAU was the first study to evaluate the effect of ADA on RA-related WPAI as well as the association between changes in disease activity and outcomes in routine rheumatology care in Japan. Results demonstrated that treatment with ADA significantly improved remission rates as defined by several disease activity measures (DAS28CRP, SDAI, and HAQ-DI scores), and thereby may have improved work productivity (including absenteeism, presenteeism, and OWI) and AI over 48 weeks. Correlation analysis showed a trend toward a positive linear correlation between improvement in WPAI/RA domain scores and decrease in disease activity at week 48 , except for absenteeism in PTWs. The more prominent improvement in work productivity was noted in PWs compared with PTWs. In general, despite differences in work culture and baseline work

SDAI Simplified Disease Activity Index (to assess functional response). ${ }^{*} p<0.0001$; the Fisher exact test was used to compare remission rate at baseline and at 12,24, and 48 weeks. Data were analyzed using the last observation carried forward method. The Wilcoxon signed-rank test was used to compare EQ-5D-3L at baseline and at 12, 24, and 48 weeks

productivity scores, these results are consistent with findings from studies evaluating the effect of ADA on work and household productivity in other countries $[19,28]$. Treatment with ADA also resulted in substantial improvements in health-related QoL (as assessed by EQ-5D-3L scores) in PWs and HMs. Additionally, correlation analysis suggested that improvement in WPAI domain scores is associated with better QoL.

The safety profile in this large, real-world study was consistent with previous reports, and no new safety signals were identified $[39,41]$. Serious adverse events occurred in 5.23\% of patients in this 48-week study, which is similar to the results of a previously reported ADA post-marketing surveillance study in Japan (4.5\% at week $28, n=7740$ patients) [39].

In patients with longstanding RA, evidence indicates that work disability is associated with 
Table 2 Correlations between WPAI/RA domain scores and clinical responses at 48 weeks

\begin{tabular}{|c|c|c|c|c|}
\hline \multirow[t]{2}{*}{ WPAI/RA domains } & \multicolumn{4}{|l|}{ Clinical responses } \\
\hline & $\begin{array}{l}\text { DAS28CRP } \\
r\end{array}$ & $\begin{array}{l}\text { SDAI } \\
r\end{array}$ & $\begin{array}{l}\text { HAQ-DI } \\
r\end{array}$ & $\begin{array}{l}\text { EQ-5D-3L } \\
r\end{array}$ \\
\hline \multicolumn{5}{|l|}{ PW } \\
\hline Absenteeism & $0.2138^{\mathrm{a}}$ & $0.2213^{\mathrm{a}}$ & $0.3023^{\mathrm{a}}$ & $-0.1997^{\mathrm{a}}$ \\
\hline Presenteeism & $0.4520^{\mathrm{a}}$ & $0.4235^{\mathrm{a}}$ & $0.5675^{\mathrm{a}}$ & $-0.4834^{\mathrm{a}}$ \\
\hline OWI & $0.4669^{\mathrm{a}}$ & $0.4333^{\mathrm{a}}$ & $0.5741^{\mathrm{a}}$ & $-0.4888^{\mathrm{a}}$ \\
\hline AI & $0.5159^{\mathrm{a}}$ & $0.4904^{\mathrm{a}}$ & $0.6344^{\mathrm{a}}$ & $-0.5235^{\mathrm{a}}$ \\
\hline \multicolumn{5}{|l|}{ PTW } \\
\hline Absenteeism & $0.1150(p=0.1318)$ & $0.0983(p=0.2022)$ & $0.2350^{\mathrm{b}}$ & $-0.0610(p=0.4170)$ \\
\hline Presenteeism & $0.4574^{\mathrm{a}}$ & $0.4871^{\mathrm{a}}$ & $0.5565^{\mathrm{a}}$ & $-0.3624^{\mathrm{a}}$ \\
\hline OWI & $0.4043^{c}$ & $0.4154^{\mathrm{a}}$ & $0.4950^{\mathrm{a}}$ & $-0.3290^{\mathrm{a}}$ \\
\hline AI & $0.5045^{\mathrm{a}}$ & $0.5328^{\mathrm{a}}$ & $0.6516^{\mathrm{a}}$ & $-0.4877^{\mathrm{a}}$ \\
\hline \multicolumn{5}{|l|}{ HM } \\
\hline AI & $0.4473^{\mathrm{a}}$ & $0.4327^{\mathrm{a}}$ & $0.5500^{\mathrm{a}}$ & $-0.5054^{\mathrm{a}}$ \\
\hline
\end{tabular}

For regression coefficient $(r)$, Pearson product-moment was used to examine the correlations between WPAI/RA domains (absenteeism, presenteeism, OWI, and AI) and clinical responses (DAS28CRP, SDAI, HAQ-DI, and EQ-5D-3L)

$A I$ activity impairment, CRP C-reactive protein, DAS28CRP Disease Activity Score based on 28 joints and CRP, EQ-5D-3L, EuroQol 5-Dimension 3-level, HAQ-DI Health Assessment Questionnaire-Disability Index, $H M$ homemaker, $O W I$ overall work impairment, $P T W$ part-time worker, $P W$ paid worker, $r$ regression coefficient, $R A$ rheumatoid arthritis, $S D A I$ Simplified Disease Activity Index (to assess functional response), WPAI/RA RA-related work productivity and activity impairment

${ }^{\mathrm{a}} p<0.0001$ indicates significance

b $p=0.0015$

c $p=0.0033$

disease activity and duration of RA, as well as decreased physical function [42-44]. Therefore, the importance of achieving and maintaining early remission cannot be understated. In the present study, PWs at high risk of work disability showed significantly improved WPAI outcomes after treatment with ADA, as assessed by widely used WPAI/RA domain scores (absenteeism, presenteeism, OWI, and AI) $[45,46]$. Significant decreases in DAS28CRP obtained in this real-world setting were consistent with those reported in clinical trials of 24- to 52-week duration, as well as a 24 -week post-marketing surveillance study conducted in Japan [27, 39, 47, 48]. Importantly, in the present large-scale study, improvements in
WPAI/RA showed a weak to moderately strong positive linear correlation with improvement in disease activity, extending the findings from a previous small-scale study assessing the effects of TNF- $\alpha$ antagonists on DAS28ESR and work productivity in 42 patients [15].

Presenteeism and OWI in PWs significantly improved at weeks 12 and 24 compared to PTWs (Fig. 1). At baseline, RA duration was shorter and MTX dose was higher in PWs (compared to PTWs or HMs; Table 1). Since shorter RA duration and higher MTX dose are associated with increased ADA efficacy [39], such baseline differences may in part explain better outcomes in WPAI scores (especially in presenteeism and OWI) in PWs over PTWs. 


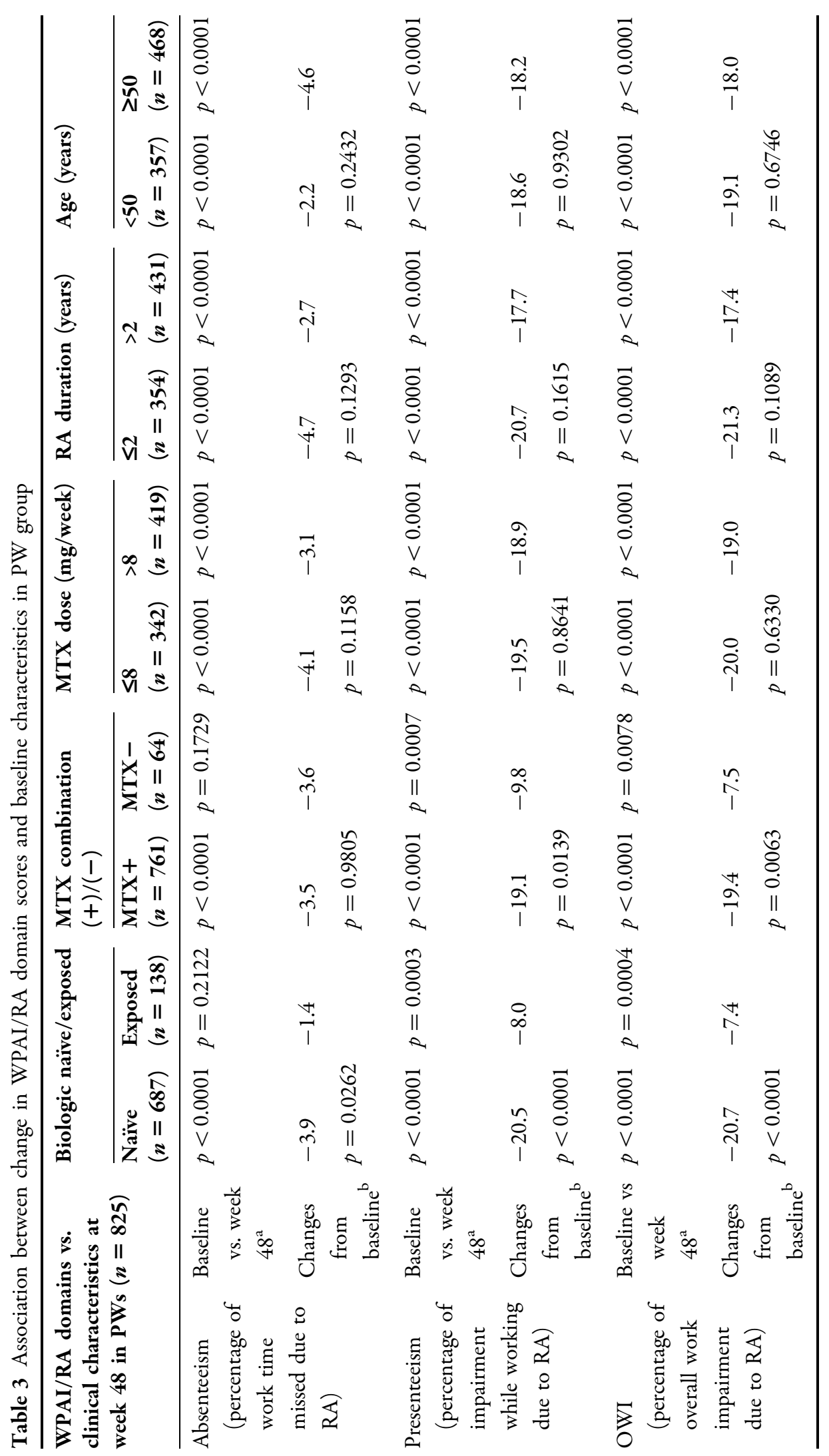




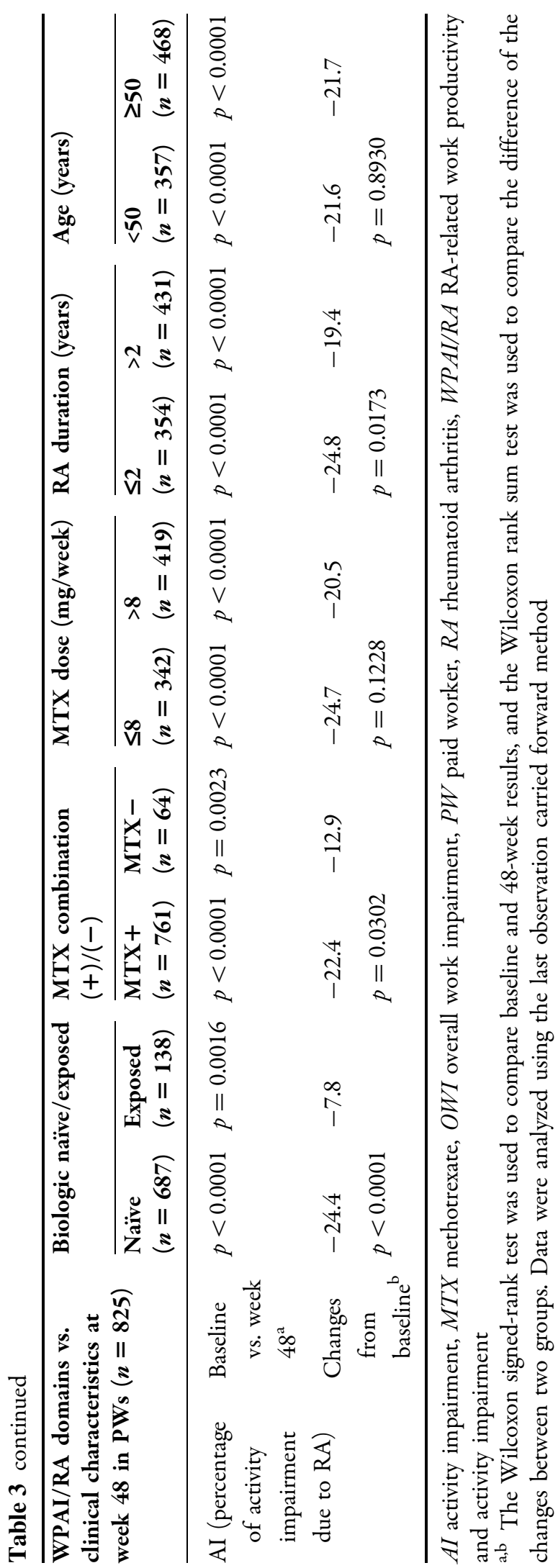

In our evaluation of the relationship between baseline characteristics of PWs and WPAI/RA, we identified biologic-naïve status as a factor associated with improvement in all aspects of work productivity, including absenteeism, presenteeism, and OWI, as well as AI. Use of biologics has been shown to reduce absenteeism and improve presenteeism in previous studies $[4,49]$. In the present study, estimated missed work days per year in PWs decreased significantly from $13.34 \pm 38.89$ days at baseline to $5.35 \pm 23.44$ days at week 48 . These results are within the range presented in a systematic literature review evaluating randomized controlled trials showing the effect of biologics on the reduction in days of sick leave (ranging from 2.1 days in 4 weeks to 18.7 days in 2 years) [49]. A favorable effect of biologics on daily work-related productivity has been reported in Japan [15]. Concomitant use of MTX was also a factor associated with presenteeism, OWI, and AI, and shorter disease duration was another factor associated with AI for HMs in the present study. These factors were also identified as contributing to the effectiveness of ADA in the 24-week post-marketing surveillance study conducted in Japan. All subgroups in that study showed significant improvement, particularly biologic-naïve patients and those receiving concomitant MTX [39, 41]. Even in Western countries, treatment with ADA + MTX has been shown to reduce the risk of work impairment as exhibited by the improvement in presenteeism, OWI, and AI scores [50].

Although RA is known to be associated with increased cardiovascular morbidity and mortality [51], anti-TNF- $\alpha$ drugs have been suggested to reduce the cardiovascular burden in RA, possibly due to their potent anti-inflammatory effects [52]. The low incidence of cardiac disorders in the present study (one case) is consistent with these prior studies, although other biologics, such as abatacept (a selective inhibitor of $\mathrm{T}$ cell co-stimulation), may have a greater impact on decreasing cardiovascular risk [53].

This was a large, real-world study; nevertheless, it had some limitations. The 
Table 4 Safety

\begin{tabular}{|c|c|c|}
\hline Serious adverse event (MedDRA system organ class) ${ }^{a}$ & Number of cases & Number of events (\%) \\
\hline Infections and infestations & 31 & $37(1.9)$ \\
\hline Respiratory, thoracic, and mediastinal disorders & 11 & $14(0.7)$ \\
\hline Neoplasms benign, malignant, and unspecified (including cysts and polyps) & 14 & $17(0.9)$ \\
\hline General disorders and administration site conditions & 4 & $5(0.3)$ \\
\hline Gastrointestinal disorders & 3 & $3(0.2)$ \\
\hline Hepatobiliary system disorders & 4 & $6(0.3)$ \\
\hline Injury, poisoning, and procedural complications & 12 & $12(0.6)$ \\
\hline Skin and subcutaneous tissue disorders & 3 & $4(0.2)$ \\
\hline Ocular disorders & 4 & $4(0.2)$ \\
\hline Musculoskeletal and connective tissue disorders & 2 & $2(0.1)$ \\
\hline Blood and lymphatic system disorders & 2 & $2(0.1)$ \\
\hline Vascular disorders & 2 & $2(0.1)$ \\
\hline Ear and labyrinth disorders & 1 & $1(0.1)$ \\
\hline Cardiac disorders & 1 & $1(0.1)$ \\
\hline Nervous system disorders & 6 & $6(0.3)$ \\
\hline Psychiatric disorders & 1 & $1(0.1)$ \\
\hline Reproductive and breast disorders & 1 & $1(0.1)$ \\
\hline Clinical laboratory test & 1 & $1(0.1)$ \\
\hline
\end{tabular}

MedDRA Medical Dictionary for Regulatory Activities

a Total serious adverse event incidence rate: $5.23 \%$ (103/1968 cases)

differences in baseline HAQ-DI, EQ-5D-3L, and AI scores could be attributed to activity associated with employment status, as the dose of prednisolone, clinical disease activity measured by DAS28ESR, and SDAI were comparable at baseline between groups. Additionally, baseline characteristics of the patient population in this real-world study were heterogeneous in comparison to those of clinical trials in Japan $[23,26,27,39$, 47, 48, 54]. Moreover, this study did not evaluate patients on the basis of comorbidity [54] and stage of disease, which could potentially impact the effectiveness of ADA.

\section{CONCLUSIONS}

ADA demonstrated significant, sustained improvements in RA-related WPAI in Japanese patients with RA. Further studies are needed to evaluate whether improved WPAI/RA scores are associated with a decreased risk of potential unemployment and financial impairment.

\section{ACKNOWLEDGEMENTS}

Sponsorship for this study and article processing charges was funded by AbbVie GK, Tokyo, Japan, and Eisai Co., Ltd., Tokyo, Japan. 
All named authors meet the International Committee of Medical Journal Editors (ICMJE) criteria for authorship for this manuscript, take responsibility for the integrity of the work as a whole, and have given final approval for the version to be published. Editorial support in the preparation of this manuscript was provided by Cactus Communications. Support for this assistance was funded by AbbVie GK and Eisai Co., Ltd., Tokyo, Japan. We also express our sincere thanks to the patients, investigators, fellows, nurses, and research coordinators at each medical institution who participated in this study. We thank EPS, Co. Ltd. and Naoto Hirota of Stella Co., Ltd. for their dedicated support in the development of the statistics plan and interpretation of the results, and Sarina Kurimoto, MD, PhD, of AbbVie GK for editorial assistance. All authors had full access to all of the data in this study and take complete responsibility for the integrity of the data and accuracy of the data analysis.

Disclosures. This study was funded by AbbVie (NCT01346488) and Eisai. Yoshiya Tanaka received research grants from Mitsubishi Tanabe, Takeda, Daiichi Sankyo, Chugai, Bristol-Myers, MSD, Astellas, AbbVie, and Eisai; consulting fees from AbbVie, Chugai, Daiichi Sankyo, Bristol-Myers, Mitsubishi Tanabe, Astellas, Takeda, Pfizer, Teijin, Asahi-kasei, YL Biologics, Sanofi, Janssen, Eli Lilly, and GlaxoSmithKline; and is a member of the speakers bureau for AbbVie, Chugai, Daiichi Sankyo, Bristol-Myers, Mitsubishi Tanabe, Astellas, Takeda, Pfizer, Teijin, Asahi-kasei, YL Biologics, Sanofi, Janssen, Eli Lilly, and GlaxoSmithKline. Naoki Agata holds stocks and stock options with AbbVie and is a full-time employee of AbbVie GK. Ataru Igarashi received research grants from Pfizer Japan Inc., CSL Behring Japan Inc., Gilead Science K.K., and Fuji Film K.K.; consulting fees from Novartis Pharma K.K., AbbVie GK, Milliman Inc., Sony Inc., and Eli Lilly Japan K.K.; and is a member of the speakers bureau for Chugai Pharmaceutical Co. Ltd., CRECON Research and Consulting Inc., Terumo Corporation, Bristol-Myers Squibb K.K., and
Creativ-Ceutical K.K. Toshiro Tango received consulting fees from AbbVie GK, Ajinomoto Pharma, Takeda Pharmaceutical Co. Ltd., and Lion Corporation. Tsutomu Takeuchi received research grants from AbbVie, Astellas, Bristol-Myers, Chugai, Daiichi Sankyo, Eisai, Janssen, Mitsubishi Tanabe, Nippon Shinyaku, Pfizer, Sanofi, Santen, Takeda, and Teijin; consulting fees from AstraZeneca, Eli Lilly, Novartis, Mitsubishi Tanabe, and Asahi Kasei Medical; and is a member of the speakers bureau for AbbVie, Bristol-Myers Squibb, Chugai, Eisai, Janssen, Mitsubishi Tanabe, Pfizer, and Takeda. Ryo Nakajima is a full-time employee of AbbVie GK. Shuichi Komatsu is a full-time employee of AbbVie GK. Kiyotaka Yamazaki is a full-time employee of AbbVie GK. Tomohiro Nakamura is a full-time employee of AbbVie GK.

Compliance with Ethics Guidelines. This study was a multi-institutional, prospective, noninterventional, observational study that conformed to the Good Post-marketing Study Practice (GPSP; Ministry of Health, Labour and Welfare ordinance). The study protocol was reviewed and approved in advance by the Pharmaceuticals and Medical Devices Agency, Japan. For this reason, no ethical review by the individual facilities participating in the study was conducted. Because informed consent is not required for post-marketing observational studies that are conducted under the GPSP in Japan, the present study did not solicit informed consent from the patients.

Data Availability. The datasets used and/or analyzed during the current study are available from the corresponding author on reasonable request.

Open Access. This article is distributed under the terms of the Creative Commons Attribution-NonCommercial 4.0 International License (http://creativecommons.org/licenses/ by-nc/4.0/), which permits any noncommercial use, distribution, and reproduction in any medium, provided you give appropriate credit to the original author(s) and the source, provide a link to the Creative Commons license, and indicate if changes were made. 


\section{REFERENCES}

1. Tanaka Y. Current concepts in the management of rheumatoid arthritis. Korean J Intern Med. 2016;31:210-8.

2. Cross M, Smith E, Hoy D, et al. The global burden of rheumatoid arthritis: estimates from the global burden of disease 2010 study. Ann Rheum Dis. 2014;73:1316-22.

3. Yamanaka H, Sugiyama N, Inoue E, Taniguchi A, Momohara S. Estimates of the prevalence of and current treatment practices for rheumatoid arthritis in Japan using reimbursement data from health insurance societies and the IORRA cohort (I). Mod Rheumatol. 2014;24:33-40.

4. Verstappen SM. Rheumatoid arthritis and work: the impact of rheumatoid arthritis on absenteeism and presenteeism. Best Pract Res Clin Rheumatol. 2015;29:495-511.

5. Bertin P, Fagnani F, Duburcq A, et al. Impact of rheumatoid arthritis on career progression, productivity, and employability: the PRET study. Jt Bone Spine. 2016;83:47-52.

6. van Vilsteren $\mathrm{M}$, Boot $\mathrm{CR}$, Knol DL, et al. Productivity at work and quality of life in patients with rheumatoid arthritis. BMC Musculoskelet Disord. 2015;16:107.

7. Almoallim H, Kamil A. Rheumatoid arthritis: should we shift the focus from "treat to target" to "treat to work?". Clin Rheumatol. 2013;32:285-7.

8. Rheumatoid Arthritis (RA). 2016. http://www.cdc. gov/arthritis/basics/rheumatoid.htm. Accessed 7 Oct 2016.

9. Geuskens GA, Hazes JM, Barendregt PJ, Burdorf A. Predictors of sick leave and reduced productivity at work among persons with early inflammatory joint conditions. Scand J Work Environ Health. 2008;34:420-9.

10. Hoshi D, Tanaka E, Igarashi A, et al. Profiles of EQ-5D utility scores in the daily practice of Japanese patients with rheumatoid arthritis; analysis of the IORRA database. Mod Rheumatol. 2016;26:40-5.

11. Tanaka E, Hoshi D, Igarashi A, et al. Analysis of direct medical and nonmedical costs for care of rheumatoid arthritis patients using the large cohort database, IORRA. Mod Rheumatol. 2013;23:742-51.

12. Bansback N, Zhang W, Walsh D, et al. Factors associated with absenteeism, presenteeism and activity impairment in patients in the first years of RA. Rheumatology. 2012;51:375-84.

13. Singh JA, Furst DE, Bharat A, et al. 2012 update of the 2008 American College of Rheumatology recommendations for the use of disease-modifying antirheumatic drugs and biologic agents in the treatment of rheumatoid arthritis. Arthritis Care Res. 2012;64:625-39.

14. Smolen JS, Aletaha D, Bijlsma JW, et al. Treating rheumatoid arthritis to target: recommendations of an international task force. Ann Rheum Dis. 2010;69:631-7.

15. Furuya H, Kasama T, Isozaki T, et al. Effect of TNF antagonists on the productivity of daily work of patients with rheumatoid arthritis. J Multidiscip Healthc. 2013;6:25-30.

16. Smolen JS, Han C, van der Heijde D, et al. Infliximab treatment maintains employability in patients with early rheumatoid arthritis. Arthritis Rheum. 2006;54:716-22.

17. Keat AC, Gaffney K, Gilbert AK, Harris C, Leeder J. Influence of biologic therapy on return to work in people with work disability due to ankylosing spondylitis. Rheumatology. 2008;47:481-3.

18. Allaire S, Wolfe F, Niu J, Zhang Y, Zhang B, LaValley M. Evaluation of the effect of anti-tumor necrosis factor agent use on rheumatoid arthritis work disability: the jury is still out. Arthritis Rheum. 2008;59(8):1082-9.

19. Bejarano V, Quinn M, Conaghan PG, et al. Effect of the early use of the anti-tumor necrosis factor adalimumab on the prevention of job loss in patients with early rheumatoid arthritis. Arthritis Rheum. 2008;59(10):1467-74.

20. Raterman HG, Hoving JL, Nurmohamed MT, et al. Work ability: a new outcome measure in rheumatoid arthritis? Scand J Rheum. 2010;39:127-31.

21. Prescribing information. 2016. http://www. rxabbvie.com/pdf/humira.pdf. Accessed 7 Oct 2016.

22. Miyasaka N. Adalimumab for the treatment of rheumatoid arthritis. Expert Rev Clin Immunol. 2009;5:19-26.

23. Yamanaka $\mathrm{H}$, Ishiguro $\mathrm{N}$, Takeuchi $\mathrm{T}$, et al. Recovery of clinical but not radiographic outcomes by the delayed addition of adalimumab to methotrexate-treated Japanese patients with early rheumatoid arthritis: 52-week results of the HOPEFUL-1 trial. Rheumatology. 2014;53:904-13. 
24. Smolen JS, Emery P, Fleischmann R, et al. Adjustment of therapy in rheumatoid arthritis on the basis of achievement of stable low disease activity with adalimumab plus methotrexate or methotrexate alone: the randomised controlled OPTIMA trial. Lancet. 2014;25(383):321-32.

25. Takeuchi $\mathrm{T}$, Yamanaka $\mathrm{H}$, Ishiguro $\mathrm{N}$, et al. Adalimumab, a human anti-TNF monoclonal antibody, outcome study for the prevention of joint damage in Japanese patients with early rheumatoid arthritis: the HOPEFUL 1 study. Ann Rheum Dis. 2014;73:536-43.

26. Tanaka $\mathrm{Y}$, Yamanaka $\mathrm{H}$, Ishiguro $\mathrm{N}$, et al. Adalimumab discontinuation in patients with early rheumatoid arthritis who were initially treated with methotrexate alone or in combination with adalimumab: 1 year outcomes of the HOPEFUL-2 study. RMD Open. 2016;2:e000189.

27. Kimura N, Suzuki K, Takeuchi T. Time lag between the initiation of adalimumab after methotrexate correlates with the efficacy of adalimumab in rheumatoid arthritis patients. Mod Rheumatol. 2016;26:676-80.

28. Hussain W, Janoudi N, Noorwali A, et al. Effect of Adalimumab on Work Ability Assessed in Rheumatoid Arthritis Disease Patients in Saudi Arabia (AWARDS). Open Rheumatol J. 2015;9:46-50.

29. van Vollenhoven RF, Cifaldi MA, Ray S, Chen N, Weisman $\mathrm{MH}$. Improvement in work place and household productivity for patients with early rheumatoid arthritis treated with adalimumab plus methotrexate: work outcomes and their correlations with clinical and radiographic measures from a randomized controlled trial companion study. Arthritis Care Res. 2010;62:226-34.

30. Halpern MT, Cifaldi MA, Kvien TK. Impact of adalimumab on work participation in rheumatoid arthritis: comparison of an open-label extension study and a registry-based control group. Ann Rheum Dis. 2009;68:930-7.

31. Zhang W, Bansback N, Guh D, et al. Short-term influence of adalimumab on work productivity outcomes in patients with rheumatoid arthritis. J Rheumatol. 2008;35:1729-36.

32. Mittendorf T, Dietz B, Sterz R, Cifaldi MA, Kupper $\mathrm{H}$, von der Schulenburg JM. Personal and economic burden of late-stage rheumatoid arthritis among patients treated with adalimumab: an evaluation from a patient's perspective. Rheumatology. 2008;47:188-93.
33. Tanaka E, Inoue E, Mannalithara A, et al. Medical care costs of patients with rheumatoid arthritis during the prebiologics period in Japan: a large prospective observational cohort study. Mod Rheumatol. 2010;20:46-53.

34. Japan College of Rheumatology. Guidelines for adalimumab (in Japanese). 2008. http://www. ryumachi-jp.com/info/guideline_ADA.pdf. Accessed 7 Oct 2016.

35. HUMIRA (adalimumab) [prescribing information]. Pharmaceuticals and medical devices agency Web site [In Japanese]. http://www.pmda.go.jp/ PmdaSearch/iyakuDetail/ResultDataSetPDF/ 112130_3999426G1024_2_10. Accessed 7 Oct 2016.

36. Koike R, Takeuchi T, Eguchi K, Miyasaka N. Update on the Japanese guidelines for the use of infliximab and etanercept in rheumatoid arthritis. Mod Rheumatol. 2007;17:451-8.

37. Tony HP, Hein G, Richter C, et al. Adalimumab: Wirksamkeit und Sicherheit bei Patienten mit rheumatoider Arthritis in Deutschland: Zwischenauswertung der Langzeitdokumentation HUM03-1 im Alltag der rheumatologischen Praxis mit 4600 Patienten nach 24 Monaten Behandlung. Zeitschrift für Rheumatol. 2007;60(Suppl. 1):S42.05.

38. Steinbrocker $\mathrm{O}$, Traeger $\mathrm{CH}$, Batterman RC. Therapeutic criteria in rheumatoid arthritis. J Am Med Assoc. 1949;140(8):659-62.

39. Koike T, Harigai M, Ishiguro N, et al. Safety and effectiveness of adalimumab in Japanese rheumatoid arthritis patients: postmarketing surveillance report of the first 7740 patients. Mod Rheumatol. 2014;24:390-841.

40. Koike T, Harigai M, Ishiguro N, et al. Safety and effectiveness of adalimumab in Japanese rheumatoid arthritis patients: postmarketing surveillance report of the first 3000 patients. Mod Rheumatol. 2012;22:498-508.

41. Pincus T, Callahan LF, Sale WG, Brooks AL, Payne LE, Vaughn WK. Severe functional declines, work disability, and increased mortality in seventy-five rheumatoid arthritis patients studied over nine years. Arthritis Rheum. 1984;27:864-72.

42. Yelin E, Henke C, Epstein W. The work dynamics of the person with rheumatoid arthritis. Arthritis Rheum. 1987;30:507-12.

43. Wolfe F, Hawley DJ. The longterm outcomes of rheumatoid arthritis: Work disability: a prospective 18 year study of 823 patients. J Rheumatol. 1998;25:2108-17. 
44. Langley PC, Mu R, Wu M, Dong P, Tang B. The impact of rheumatoid arthritis on the burden of disease in urban China. J Med Econ. 2011;14:709-19.

45. Zhang W, Bansback N, Kopec J, Anis AH. Measuring time input loss among patients with rheumatoid arthritis: validity and reliability of the Valuation of Lost Productivity questionnaire. J Occup Environ Med. 2011;53:530-6.

46. Kaneko A, Hirano Y, Fujibayashi T, et al. Twenty-four-week clinical results of adalimumab therapy in Japanese patients with rheumatoid arthritis: retrospective analysis for the best use of adalimumab in daily practice. Mod Rheumatol. 2013;23:466-77.

47. Takeuchi T, Tanaka Y, Kaneko Y, et al. Effectiveness and safety of adalimumab in Japanese patients with rheumatoid arthritis: retrospective analyses of data collected during the first year of adalimumab treatment in routine clinical practice (HARMONY study). Mod Rheumatol. 2012;22:327-38.

48. ter Wee MM, Lems WF, Usan H, Gulpen A, Boonen A. The effect of biological agents on work participation in rheumatoid arthritis patients: a systematic review. Ann Rheum Dis. 2012;71:161-71.

49. Emery P, Smolen JS, Ganguli A, et al. Effect of adalimumab on the work-related outcomes scores in patients with early rheumatoid arthritis receiving methotrexate. Rheumatology. 2016;55(8):1458-65.

50. Lazurova I, Tomas L. Cardiac impairment in rheumatoid arthritis and influence of anti-TNFalpha treatment. Clin Rev Allergy Immunol. 2016. doi:10.1007/s12016-016-8566-3.

51. Cugno M, Ingegnoli F, Gualtierotti R, Fantini F. Potential effect of anti-tumour necrosis factor-alpha treatment on reducing the cardiovascular risk related to rheumatoid arthritis. Curr Vasc Pharmacol. 2010;8:285-92.

52. Zhang J, Xie F, Yun H, et al. Comparative effects of biologics on cardiovascular risk among older patients with rheumatoid arthritis. Ann Rheum Dis. 2016;75(10):1813-8.

53. Oh $\mathrm{K}$, Ito $\mathrm{S}$, Unno $\mathrm{M}$, et al. The rate of decrease in the disease activity of rheumatoid arthritis during treatment with adalimumab depends on the dose of methotrexate. Intern Med. 2015;54:1035-41.

54. Nakajima A, Inoue E, Shimizu Y, et al. Presence of comorbidity affects both treatment strategies and outcomes in disease activity, physical function, and quality of life in patients with rheumatoid arthritis. Clin Rheumatol. 2015;34:441-9. 